\title{
Emerging Technologies in Electrophysiology: From Single-Chamber to Biventricular Leadless Pacemakers
}

\author{
Jashan Gill ${ }^{a, b}$ \\ aDepartment of Medicine, Rosalind Franklin University of Medicine and Science, North Chicago, IL, USA; \\ ${ }^{b}$ Department of Medicine, Northwestern Medicine McHenry Hospital, McHenry, IL, USA
}

\section{Keywords}

Nanostim - WiSE cardiac resynchronization therapy .

Subcutaneous implantable cardioverter-defibrillator .

Leadless cardiac resynchronization therapy · Arrhythmia

\begin{abstract}
Background: Transvenous pacemakers have been shown to improve quality of life and mortality in patients with bradycardia and cardiac conduction blocks. However, they possess inevitable drawbacks as they have a relatively high incidence of lead and device pocket-related complications. Therefore, leadless pacemakers have emerged as a solution to reduce the complications seen with conventional pacemakers. However, there have been no clinical trials to date comparing transvenous to leadless pacemakers. Summary: Currently, the Micra ${ }^{\mathrm{TM}}$ transcatheter pacing system or AV device has been approved for commercial use worldwide but is limited to single-chamber pacing with single- or dualchamber sensing. Although the leadless pacemaker Nanostim $^{\mathrm{Tm}}$ was initially promising, it has been recalled due to concerns of battery failures and is no longer approved in Europe. In addition, the lack of defibrillation capabilities with leadless pacemakers has been a limiting factor; therefore, a leadless pacemaker with the already approved subcutaneous cardioverter-defibrillator system is currently being studied in humans. Moreover, the WiSE cardiac resynchronization
\end{abstract}

Karger@karger.com

(c) 2022 S. Karger AG, Basel

www.karger.com/crd

Karger! therapy (CRT) device has been approved in Europe, with the capabilities for leadless CRT in patients with unsuitable coronary sinus anatomy. Furthermore, retrieval of leadless pacemakers has been an area of concern; however, clinic data have signaled toward safe extraction of these devices with minimal complications. Key Messages: This review will encompass the current literature regarding clinical safety and outcomes of these novel leadless pacemakers and discuss the evolving technologies in the field of cardiac pacing.

(c) 2022 S. Karger AG, Basel

\section{Introduction}

It is estimated that more than 700,000 new pacemakers were implanted in 2009 [1]; in recent years, these rates have exponentially increased as greater than 1 million devices are implanted annually [2]. Since their introduction in 1958 [3], transvenous pacemakers have improved quality of life and reduced mortality in patients with bradyarrhythmia or heart block [4-6]. Despite proving to be lifesaving, these devices still possess significant limitations, with reported complication rates of $8-12 \%$ in the short- and long-term settings $[7,8]$. Complications are typically related to the transvenous leads and subcutaneous device pockets. These consist of pocket hematoma, pneumothorax, cardiac tamponade, lead dislodgement, 


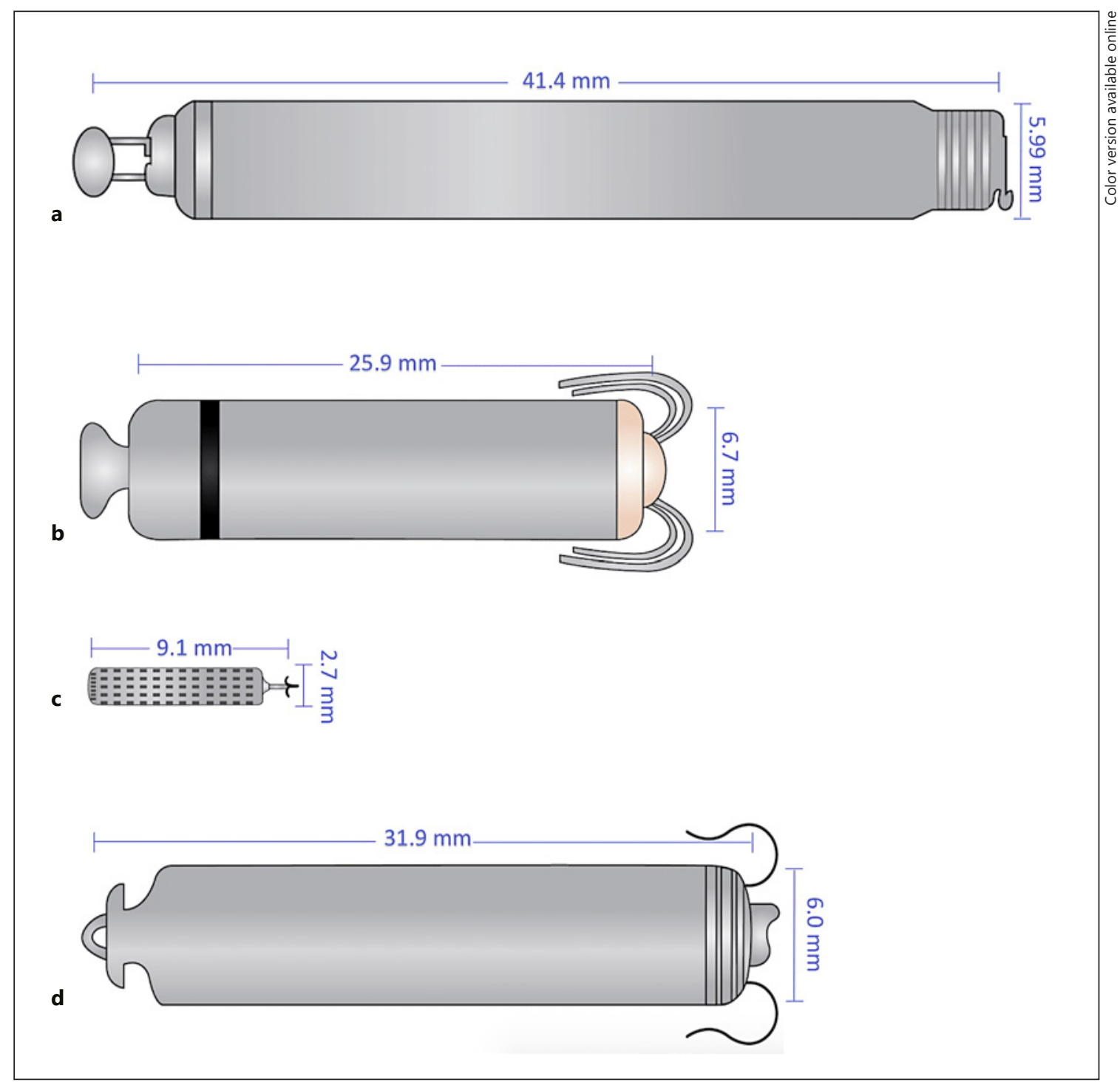

Fig. 1. Leadless Pacemakers. Illustration of leadless pacemakers, $\operatorname{Nanostim}^{\mathrm{TM}}(\mathbf{a}), \operatorname{Micra}^{\mathrm{TM}}(\mathbf{b})$, the left ventricular electrode in the WiSE-CRT ${ }^{\mathrm{TM}}$ system $(\mathbf{c})$, and $\mathrm{EMPOWER}^{\mathrm{TM}}(\mathbf{d})$ with relative size comparisons and accurate measurements.

venous obstruction, systemic infection, or endocarditis [9-13]. Pocket and transvenous lead-related infections are particularly severe, with an associated mortality of up to $12 \%$ and $31 \%$, respectively, requiring removal of all hardware [14]. Moreover, leads may also fracture from mechanical trauma, causing malfunction and insulation disruptions, resulting in abnormal pacing and sensory function $[15,16]$. Therefore, reducing pacemaker hardware is essential to mitigate these complications.

The concept of a leadless pacemaker that could overcome the limitations of transvenous pacers was first pro- posed in 1970 by Spickler et al. [17]. In the ensuing years, several studies were conducted in animals, demonstrating the feasibility, efficacy, and safety of leadless pacemakers [18-21]. With technological advances in transcatheter interventions, device miniaturization, communications, and battery longevity, this concept became realistic in humans and has resulted in a paradigm shift in modern medicine. This review will summarize the clinical safety and efficacy of the current leadless devices (Fig. 1) and discuss the emerging technologies in this rapidly advancing field. 
Table 1. Comparison of leadless devices

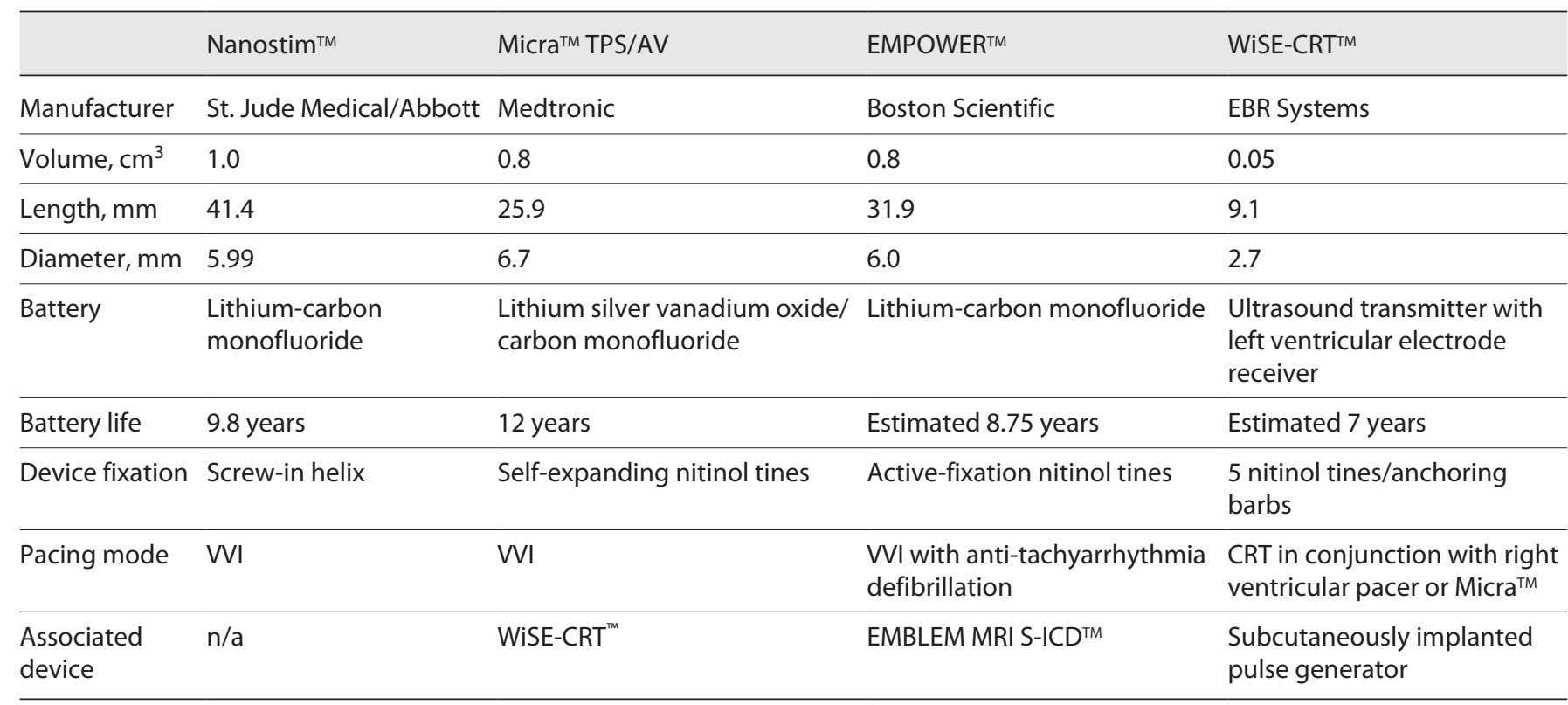

\section{Leadless Single-Chamber Ventricular Pacemakers}

Currently, two leadless devices have been developed with ventricular pacing, sensing, and inhibition pacing (VVI) capabilities. These are Nanostim ${ }^{\mathrm{TM}}$, manufactured by St. Jude Medical and Micra ${ }^{\mathrm{TM}}$ transcatheter pacing system (TPS), manufactured by Medtronic. The design of these devices is similar as they are entirely self-contained, including pacemaker electronics, lithium batteries, and electrodes. Both are delivered to the right ventricle under fluoroscopic guidance via a deflectable catheter with a sheath system from the femoral vein. Minor differences lie in implantation; Nanostim ${ }^{\mathrm{TM}}$ is secured by a single turn (screw-in) into the endocardium with a maximum depth of $1.3 \mathrm{~mm}$ [22]. Micra ${ }^{\mathrm{TM}}$ TPS is fixated to the endocardium by four nitinol tines. Both devices are connected to the catheter by tethers used to confirm adequate fixation with a tug test before the device is released [23]. Both devices allow response rate programming via a temperature sensor (Nanostim ${ }^{\mathrm{TM}}$ ) or accelerometer (Micra ${ }^{\mathrm{TM}} \mathrm{TPS}$ ) [24]. Details and comparisons between these devices are displayed in Table 1 . These devices are only capable of VVI pacing, lacking defibrillation capacity, which limits their utility. Currently, they are suitable for patients with permanent atrial fibrillation with a slow ventricular response, bradycardia-tachycardia syndrome in the setting of sick sinus syndrome, atrioventricular (AV) or sinus node block in which frequent ventricular pacing is not

Leadless Pacemakers: Current and Future Devices expected, and recurrent syncope due to vagally induced cardio-inhibition [25]. MRI compatibility is also an important consideration; both devices are MRI-conditional. Micra $^{\mathrm{TM}}$ TPS is safe for $1.5 \mathrm{~T}$ and 3.0T scanners [26, 27]; however, Nanostim ${ }^{\mathrm{TM}}$ is only proven safe for $1.5 \mathrm{~T}$ scanners [28].

\section{Clinical Safety and Efficacy}

\section{Nanostim $^{T M}$}

The landmark LEADLESS trial [22] was the first-inman study assessing the feasibility of a leadless pacemaker. This was a multicenter trial conduction in Europe, enrolling 33 patients with a primary safety endpoint defined as freedom from device or implant-related complications at 90 days. As part of secondary performance endpoints, device performance measures (sensing, impedance, Rwave amplitude, and pacing threshold) were also evaluated. In this trial, 32 out of 33 (97\%) devices were successfully implanted, and the primary safety endpoint was met as 31 out of $33(94 \%)$ participants were complication-free. Of the 2 complications, 1 patient had a perforation of the right ventricular apex resulting in cardiac tamponade and eventual death. The second patient had incorrect device implantation into the left ventricle (LV) through a patent foramen ovale. The device was successfully retrieved and correctly place, and the patient did not experience any 
further adverse outcomes [22]. In this study, Nanostim ${ }^{\mathrm{TM}}$ proved to be safe and efficacious without battery complications and sensing or capturing malfunctions. Therefore, a CE mark for European approval was granted in October 2013. At 1-year follow-up, the results of this trial were sustained in the 31-patient cohort as there was stable device performance (pacing threshold, sensing amplitude, and impedance) with no reported device-related complications [29]. The LEADLESS II IDE (FDA investigational device exemption) [30] was the second trial with Nanostim ${ }^{\mathrm{TM}}$ conducted across 3 countries (the USA, Canada, Australia), assessing clinical safety and efficacy. The pacemaker was implanted successfully in 504 out of the 526 participants $(95.8 \%), 300$ of which met the minimum follow-up of 6 months. The primary efficacy endpoint of an appropriate pacing capture threshold $(\leq 2.0 \mathrm{~V}$ at $0.4 \mathrm{~ms}$ ) and R-wave amplitude (R-wave $\geq 5.0 \mathrm{mV}$ or equal or greater than the value at implantation) were met in $90 \%$ of patients ( 270 out of the 300 ). The primary safety endpoint (freedom from device-related serious adverse events at 6 months) was met at 93\% (280 out of the 300 patients). Twenty-two serious adverse events occurred in $20(6.7 \%)$ of the participants. These events included device dislodgement requiring percutaneous retrieval $(1.7 \%)$, cardiac perforation (1.3\%), pacing threshold elevation requiring replacement (1.3\%), and vascular complications (1.3\%). Device dislodgement occurred 4 times to the pulmonary artery and twice to the right femoral vein, irrespective of implantation site. However, in totality, these results were again favorable as patients saw an improved mean R-wave amplitude from $7.8 \pm 2.9$ to 9.2 $\pm 2.9(p<0.01)$, increased ventricular pacing, and pacing capture threshold at 0.4 milliseconds $(\mathrm{ms})$ decreased from $0.82 \pm 0.69 \mathrm{~V}$ to $0.58 \pm 0.31 \mathrm{~V}(p<0.01)$ at 1 -year follow-up [30].

The LEADLESS Observational Study was a post-marketing trial conducted primarily in Europe, evaluating the short- and long-term safety of Nanostim ${ }^{\mathrm{TM}}$ in the realworld setting [31]. The primary endpoint of this study was an evaluation of safety as freedom from serious adverse device events (SADE) at 180 days in 300 subjects. Incidents of cardiac perforation resulting in death occurred in 2 out of the 131 initial implants, leading to trial suspension in April 2014. An analysis of events identified a learning curve with device deployment; therefore, additional training and updated protocols were instituted. The trial was resumed after these protocol changes; the subsequent freedom from SADE was $94.6 \%$. A total of 18 SADE occurred in 16 patients; the majority of these events (88.9\%) occurred within 30 days of implantation. There were 8 cardiac perforations, 1 device dislodgement to the right pulmonary artery, and 2 vascular complications. Similar to previous trials, pacing capture thresholds of Nanostim ${ }^{\mathrm{TM}}$ were consistent with traditional pacemakers at 6 months. However, in October 2016, St. Jude Medical issued a stop advisory, and implants were halted worldwide after reports of battery failures resulting in loss of pacing output and communications. After the LEADLESS observational trial's conclusion date, 19 battery failures were reported [31]. These concerns prompted the replacement of 1423 Nanostim $^{\mathrm{TM}}$ devices with either a transvenous pacemaker or Micra ${ }^{\mathrm{TM}}$ device. Of all the implanted devices worldwide, 34 battery failures (2.4\%) were identified (Europe $=30$, USA $=3$, and Australia $=$ 1 ), occurring at an average of 3 years. Analysis of the retrieved device indicated reduced electrolyte within the lithium-carbon monofluoride battery, causing increased battery resistance and loss of adequate current for device functionality [32]. Moreover, an observational singlecenter study consisting of 14 patients with a mean followup of 33.3 months reported an alarmingly high incidence of battery failure at $40 \%$ after 3 years [33]. The increased percentage of failure in this study was likely because of the longer follow-up in these patients compared to the initial trials as battery failure was time-dependent. Currently, Abbott has pivoted away from Nanostim ${ }^{\mathrm{TM}}$ to a new ventricular device called Aveir ${ }^{\mathrm{TM}}$ leadless pacemaker system. Safety and efficacy will be evaluated in the LEADLESS II IDE study (phase II), which is currently ongoing [34].

Micra $^{T M}$ TPS

Preclinical trials with Micra ${ }^{\mathrm{TM}}$ in animals demonstrated performance and safety similar to transvenous pacemakers [35] with accurate measurement of pacing thresholds and R-wave amplitudes [36]. The Micra TPS IDE study [37] was the first-in-man trial evaluating short- (6 months) and long-term performance (1 year) and safety of Micra ${ }^{\text {TM }}$ TPS at 70 centers worldwide. The primary safety endpoint was freedom from system-related or procedure-related major complications. The primary efficacy endpoint was defined as pacing capture threshold of $\leq 2 \mathrm{~V}$ at $0.24 \mathrm{~ms}$ at 6 months, without an increase by more than $1.5 \mathrm{~V}$ from implantation time [38]. The device was successfully placed in 719 of 725 participants (99.2\%). Both safety and efficacy endpoints were met as adverse events occurred in only $4 \%$ of participants, and pacing capture thresholds were met in $98.3 \%$ of participants. At 6 months, 28 major complications occurred in 25 patients. Notably, no device dislodgements occurred, and the major complications observed included cardiac per- 
forations $(1.5 \%)$, vascular complications $(0.7 \%)$, venous thromboembolism $(0.3 \%)$, and increased pacing thresholds (0.3\%). Moreover, a post hoc analysis comparing these 725 patients to 2,667 patients receiving transvenous pacemakers showed a reduced risk of major complications $(4.0 \%$ vs. $7.4 \%$; HR, $0.49 ; 95 \% \mathrm{CI}, 0.33-0.75, p=$ 0.001 ) [37]. At 1-year follow-up, these results were stable as freedom from the complication rate was again $96 \%$, and the efficacy endpoint was met in $93 \%$ of participants [39]. Micra ${ }^{\mathrm{TM}}$ TPS obtained CE approval in 2015 and subsequently FDA approval in 2016. The initial real-world outcomes of Micra ${ }^{\mathrm{TM}}$ were reported by the Micra TPS Post-Approval Registry consisting of 795 participants. The incidence of device-related complications was lower than reported in the Micra TPS IDE trial $(2.89 \%)$ as the 30 -day major complication rate was only $1.51 \%$. Furthermore, the device was successfully placed in 792 of 795 (99.6\%) participants. Pacing capture thresholds were also maintained at $97 \%$, consistent with results from the Micra TPS IDE trial [40]. Currently, the largest prospective study Micra CED is ongoing, consisting of a Medicare beneficiary population with an estimated to enrollment of 37,000 participants with a 2-year follow-up [41].

\section{Leadless AV Synchronization}

By virtue of the safety and efficacy of Micra ${ }^{\mathrm{TM}}$ TPS demonstrated in clinical trials, interest in expanding device capabilities emerged since VVI pacemakers only encompassed $14 \%$ of total pacemaker implantations [42]. Dual-chamber pacemakers providing AV synchrony have been shown to decrease pacemaker syndrome, improve stroke volume, and positively influence functional status and quality of life in patients with an AV block [43-45]. Therefore, 3 clinical trials, MASS, MASS II, and MARVEL, were conducted to evaluate the feasibility of an innovative AV synchronous algorithm downloaded into an implanted Micra ${ }^{\mathrm{TM}}$ device incorporating ventricularly paced, dual-chamber sensed (VDD) pacing capabilities [46]. In the MASS and MASS II studies, intracardiac accelerometer signals were obtained from the implanted $\mathrm{Micra}^{\mathrm{TM}}$, which sensed atrial contraction during various patient activities to create a sensing algorithm. This algorithm was based on 3-axis accelerometer signals collected from 4 distinct segments of the cardiac cycle corresponding to isovolumic contraction and mitral/tricuspid valve closure (A1), aortic/pulmonic valve closure (A2), passive ventricular filling (A3), and atrial contraction (A4). Blanking windows in the system rejected signals that were

Leadless Pacemakers: Current and Future Devices ventricular in origin (A1, A2), isolating atrial signals. Atrial contraction was detected when the filtered and rectified accelerometer signal exceeded a programmed threshold. The algorithm also incorporated a smoothing feature that maintained AV synchronous pacing during diminished A4 sensing, such as during patient activity [46].

The MARVEL study assessed the feasibility of the algorithm developed in MASS/MASS II studies by downloading the algorithm into patients' implanted Micra ${ }^{\mathrm{TM}}$ device for AV synchronous pacing [46]. This study demonstrated that AV synchronous pacing with the algorithm was feasible in tracking atrial contractions. The average synchronous pacing during algorithm application was $87 \%$ (95\% CI; 81.8-90.9\%). In patients with a highgrade AV block, the algorithm improved the AV synchrony percentage from $37.5 \%$ to $80 \%(p<0.001)$ compared to VVI pacing. Furthermore, left ventricular outflow velocity measured by echocardiography was significantly higher with the algorithm compared to VVI pacing (23.9 vs. $21.8 \mathrm{~cm}, p=0.004)$. Holter monitor data from study participants revealed no pauses, pacemaker-medicated tachycardia during algorithm pacing, or adverse events related to the device or algorithm [46]. The MARVEL 2 study built upon previous results as it implemented a similar downloadable accelerometer-based algorithm for VDD pacing with enhanced features, including automated programming and mode switching algorithms that could accommodate changes in the patient rhythm and activity [47]. This study enrolled 75 participants from 12 centers worldwide who were implanted with Micra $^{\mathrm{TM}}$ and had the algorithm downloaded [47]. Similar to MARVEL I, the majority of participants completed the study procedures during a single visit. The algorithm was downloaded into the patient's device at these visits, and a specialized Holter monitor capable of storing accelerometer waveforms, device markers, and electrocardiogram data was placed. Information was then collected during VVI and VDD pacing, with the participants assuming various positions and walking velocities. Results were promising as the percentage of $\mathrm{AV}$ synchronous pacing increased from an average of $26.8 \%$ during VVI pacing to $89.2 \%$ during VDD pacing. 38 out of 40 (95\%) patients had greater than $70 \%$ AV synchrony during algorithm-medicated VDD pacing compared to 0 out of 40 with VVI pacing $(p<0.001)$. AV synchrony was $89.2 \%$ at rest and $69.8 \%$ while standing [47]. Left ventricular outflow velocity measured by echocardiography was significantly increased by $1.7 \mathrm{~cm}$ during VDD pacing from a baseline average of $22.7 \mathrm{~cm}$ during VVI pacing $(p=0.002)$. Safety 
was similar to MARVEL I as no pauses, episodes of oversensing induced tachycardia, or adverse events related to the algorithm were reported during VDD pacing [47]. The enhanced algorithm proved to be superior to the initial algorithm used in MARVEL I. A key limitation in these studies was the short observation periods used as the majority of procedures were completed during a single appointment, which may not accurately reflect realworld variability. Following this study, the Micra ${ }^{\mathrm{TM}}$ AV received FDA approval in January 2020.

\section{Leadless Pacemaker with Subcutaneous Implantable Cardioverter-Defibrillator}

Although leadless pacemakers were safe and effective, they lacked defibrillation capabilities, limiting their utility in many patients. Therefore, the concept for a leadless pacemaker with a subcutaneous implantable cardioverter-defibrillator (S-ICD) was explored [48]. The S-ICD system was first introduced in 2008 and consisted of a 3-mm tripolar electrode connected to an electrically active pulse generator. The electrode is positioned $1-2 \mathrm{~cm}$ left and parallel to the sternal midline. Its distal end is positioned adjacent to the manubriosternal junction, and the proximal sensing electrode is positioned adjacent to the xiphoid process. The pulse generator is placed over the sixth rib between the anterior and midaxillary lines. The electrode contained an $8-\mathrm{cm}$ shocking coil and 2 sensing electrodes. During operation, the cardiac rhythm is detected by the sensing electrodes and the pulse generator. The system uses feature analysis and rate detection to categorize rhythm types and determine the need for defibrillation, delivered at 80 joules. This device is implanted without fluoroscopy as only anatomical landmarks are needed to guide interventionalists [49]. After demonstrating tremendous efficacy and safety for ventricular tachycardia and fibrillation [50,51], the Boston Scientific S-ICD (EMBLEM) received CE approval in 2009 and FDA approval in 2012.

Preclinical studies with a combination system, including an S-ICD and leadless VVI pacemaker in an ovine model, demonstrated successful intrabody communication applying galvanic coupling for anti-tachycardia pacing during stimulated ventricular tachycardia [48]. A larger preclinical study assessed the acute and 3-month performance of a similar system in 3 animal models (8 ovines, 5 porcine, and 27 canines). The S-ICD device used was based on the EMBLEM with updated firmware enabling communication with a leadless pacemaker called
EMPOWER, both devices from Boston Scientific. Both devices were successfully implanted in $98 \%$ of the animals, and the pacemaker exhibited appropriate VVI functionality at the 3-month follow-up. Unidirectional communication between the devices was successful in $99 \%$ of attempts, resulting in $100 \%$ anti-tachyarrhythmia pacing delivery by the system. Furthermore, pacing did not negatively impact S-ICD sensing of cardiac rhythms at rest or during simulated ventricular arrythmias [52]. Interestingly, a novel treatment strategy called modular cardiac rhythm management allows this system to be personalized for specific clinical indications. Both component devices do not need to be simultaneously implanted; if a patient only requires defibrillation, only the S-ICD can be implanted. The leadless pacemaker can then be added in the future if the patient requires pacing for bradycardia in addition to their defibrillator or vice versa. Currently, only animal studies with this system have been completed. However, the first-in-man trials are anticipated to commence in the near future [53].

\section{Leadless Cardiac Resynchronization Therapy}

Cardiac resynchronization therapy (CRT) devices have been shown to improve mortality and quality of life in patients with heart failure with reduced ejection fraction and prolonged intraventricular conduction [54-56]. However, about $30-45 \%$ of patients eligible for these devices remain untreated because of unresponsiveness to treatment or anatomical constraints with the coronary sinus lead [57-60]. EBR systems manufactured the Wireless Cardiac Stimulation System (WiCS-LV) ${ }^{\mathrm{TM}}$, which utilized transcutaneous ultrasound energy to communicate with a leadless left ventricular pacer to overcome these deficiencies. The concept for this device proved to be safe and feasible in preclinical studies [61-63]. The WiCS-LVTM system functions in harmony with a co-implanted transvenous single or biventricular pacemaker (Fig. 2). The system provides wireless pacing by transmitting acoustic ultrasound energy from a pulse transmitter implanted subcutaneously over the ribcage to an implanted receiver electrode in the LV. The LV electrode converts the acoustic energy into an electrical pacing pulse. Biventricular synchrony is accomplished by sensing pacing signals from the co-implanted right ventricular leads and immediately transmitting acoustic energy to the LV electrode [63]. Currently, the device is implanted in a 2-step process carried out on 2 consecutive days. First, the pulse transmitter is surgically implanted subcutaneously in the 


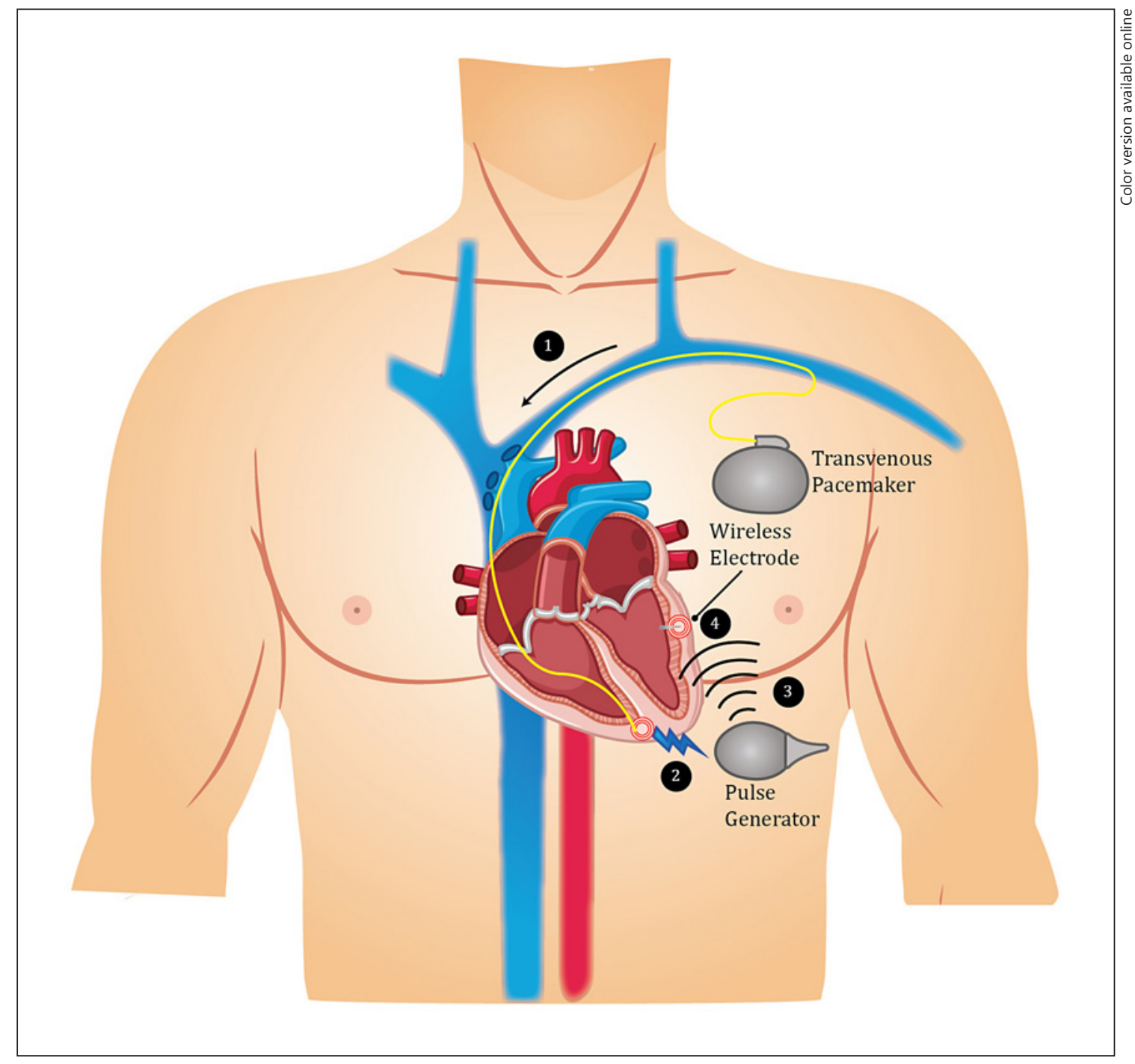

Fig. 2. Communication of the WiSE-CRT system. Illustration of the communication sequence for the WiSE-CRT device. The co-implanted transvenous pacemaker transmits a wired pacing signal to the right ventricle (\#1) detected by the subcutaneous pulse generator $(\# 2)$. The subcutaneous pulse generator transmits the signal via ultrasound energy to the left ventricular electrode (\#3), resulting in left ventricular pacing (\#4).

4th to 6th intercostal spaces lateral to the left parasternal border to deliver unimpeded acoustic signals. Second, a balloon-tipped delivery sheath containing the electrode is introduced by femoral access and advanced into the LV under fluoroscopic guidance, where the electrode is implanted. The system requires a lung- and bone-free acoustic line of sight to communicate optimally $[64,65]$.

The initial trials with this system were the WiSE-CRT [66] and the SELECT-LV [65] studies, consisting of patients unable to undergo implantation with conventional CRT devices. The WiSE-CRT study was temporarily sus- pended after 3 out of the initial 17 (18\%) implantations resulted in pericardial effusions. After review, these adverse events were noted to be caused by the catheter's delivery sheath rather than the device anchor. However, the device proved to be technologically feasible as biventricular pacing was successful in $83 \%$ of patients at 1 month and $92 \%$ at 6 months, with shorter QRS duration and improved ejection fraction in these patients [66]. Since pericardial effusions were a device limitation, the SELECTLV study featured an upgraded version of the WiCS$\mathrm{LV}^{\mathrm{TM}}$, now called WiSE-CRT ${ }^{\mathrm{TM}}$ which utilized a redesigned 
delivery system [65]. The distal portion of the delivery sheath was now equipped with a balloon to facilitate atraumatic engagement with the LV endocardium, which results in zero instances of pericardial effusions in the 35 participants. The primary performance endpoint of biventricular pacing on a 12-lead electrocardiogram was met in $97.1 \%$ (33 of 34) of participants at 1 month and 93.9\% (31 of 33) at 6 months. Although no pericardial effusions occurred, adverse events related to the device or procedure occurred in 3 participants within $24 \mathrm{~h}$ and 8 patients after 1 month. Notably, there was 1 occurrence of catheter-induced ventricular fibrillation, resulting in death and 2 occurrences of infections related to the subcutaneous pulse generator. In 1 patient, the LV electrode embolized to the left tibial artery [65]. Nonetheless, WiSE-CRT ${ }^{\mathrm{TM}}$ received CE approval after this trial. Furthermore, data from the WiSE-CRT post-market surveillance registry [67] in Europe redemonstrated the technical feasibility of the device as biventricular pacing was confirmed in $94.4 \%$ (85 of 90) of patients, and $70 \%$ had improvement in heart failure symptoms. However, the complication rate was still concerning as $4.4 \%$ experienced complications in less than $24 \mathrm{~h}, 18.8 \%$ had complications at the 30 -day follow-up, $6.7 \%$ had complications in the 1-6-month follow-up period, and 5 deaths were reported [67]. Currently, the SOLVE-CRT trial is ongoing; this is a multicenter (the USA, Europe, and Australia), randomized, double-blinded, sham-controlled trial of patients who failed or did not respond to conventional CRT with the intent to enroll 350 participants. All participants will be implanted with the WiSE-CRT ${ }^{\mathrm{TM}}$ and randomized to have the device active or turned off to evaluate the safety and performance of the device for CRT [68].

Recently, innovations have been made toward creating a totally leadless CRT system incorporating the WiSE$\mathrm{CRT}^{\mathrm{TM}}$ and Micra ${ }^{\mathrm{TM}}$ TPS devices, which was described in two published case reports $[69,70]$. The implantation procedure for each device is similar to previously described, as both devices are implanted individually. The subcutaneous pulse transmitter from the WiSE-CRT system can synchronize with the RV pacing pulse from the Micra $^{\mathrm{TM}}$ TPS and deliver a corresponding acoustic signal to the LV electrode, generating an electrical pulse within 2 ms [71]. In a 2020 observational study at 6 centers in Europe, 8 patients co-implanted with Micra ${ }^{\mathrm{TM}} \mathrm{TPS}$ and WiSE-CRT ${ }^{\mathrm{TM}}$ for CRT were assessed to demonstrate technical feasibility and safety [71]. Implantation was successful in all 8 participants without failed attempts. Seven of the 8 participants reached the 6 -month follow-up; one death occurred 4 months post-implant due to acute heart failure. Investigators noted a significant acute reduction of the QRS duration on electrocardiogram from $204.38 \pm$ $30.26 \mathrm{~ms}$ to $137.5 \pm 24.75 \mathrm{~ms}(p=0.0012)$, which was consistent at 6-month follow-up. LV ejection fraction improved post-implant by $11.29 \% \pm 8.46, p=0.018$ ). However, there was no evidence of LV reverse remodeling and nonsignificant variations in LV end-systolic and diastolic volumes [71]. This study demonstrated that both devices could successfully operate in unison to deliver CRT. However, larger prospective clinical trials are needed to represent the safety and efficacy of this system accurately. Moreover, the first case successfully implanting three coexisting systems, Micra ${ }^{\mathrm{TM}}$ TPS, WiSE-CRT ${ }^{\mathrm{TM}}$, and Emblem S-ICD, in 1 patient for CRT with defibrillation was recently published [72]. Although technically challenging, this case demonstrated the future possibilities with technological advances and additional device miniaturization. Furthermore, the combination of WiSE-CRT ${ }^{\mathrm{TM}}$ with the newly innovated Micra ${ }^{\mathrm{TM}} \mathrm{AV}$ could be a future prospect with the ability to provide VDD pacing in conjunction with CRT.

\section{Leadless Pacemaker Retrieval}

\section{Retrieval Techniques}

Although leadless pacemakers have shown promising results in clinical trials, device retrieval at the end of service, especially with chronically implanted devices, has been an area of concern. Both Nanostim ${ }^{\mathrm{TM}}$ and Micra ${ }^{\mathrm{TM}}$ are designed with unique retrieval mechanisms at their proximal ends (Fig. 1). The Nanostim ${ }^{\mathrm{TM}}$ retrieval mechanism is in the form of a docking button on its proximal end. Retrieval is facilitated with the use of an 18-French catheter with a snare of various diameters. The snare is attached to the docking button, and the protective sleeve from the catheter is advanced halfway over the device. After coaxial alignment is confirmed, the retrieval catheter is rotated counterclockwise to unscrew the device from the endocardial tissue [73].

Retrieval of $\mathrm{Micra}^{\mathrm{TM}}$ can be performed using two approaches, utilizing either the Micra ${ }^{\mathrm{TM}}$ delivery catheter (27-French) or steerable sheath. Snares of different sizes (7-10 $\mathrm{mm}$ ) and shapes (single or multiple loops) are used. Both approaches begin with advancing the delivery catheter or steerable sheath to the proximal retrieval end of the device. For the first approach, the single or triple loop snare is inserted through the central lumen of the device, and the snare is deployed around the proximal retrieval 
feature. Tension to the snare and counter traction from the cup release the tines from the endocardial tissue, and the device is withdrawn. However, engagement of the proximal retrieval feature of $\mathrm{Micra}^{\mathrm{TM}}$ is challenging. This is circumvented by the more adaptable steerable sheath used in the second approach, which allows for superior coaxial alignment. In this approach, a large caliber short sheath (11-16 French) is inserted into the Micra ${ }^{\mathrm{TM}}$ introducer sheath, preventing back bleeding. After the short sheath is inserted, the steerable sheath ( 8.5 French) is inserted into the large introducer sheath and advanced to the device. Next, a single or triple loop snare is advanced using the steerable sheath to engage the proximal retrieval feature, and traction is applied, straightening the tines and detaching the device $[74,75]$.

\section{Clinical Safety}

A small multicenter study including 16 patients implanted with Nanostim ${ }^{\mathrm{TM}}$ assessed the feasibility of shortterm retrieval at less than 6 weeks and long-term retrieval at greater than 6 weeks. The investigators noted a $100 \%$ success rate in the short-term and a 91\% long-term, without any procedure-related complications [76]. Moreover, after St Jude Medical issued a worldwide alert of battery malfunctions occurring with Nanostim ${ }^{\mathrm{TM}}$, Lakkireddy et al. [32] sought to retrieve 73 implanted devices. A total of 66 out of those devices were successfully retrieved (90.4\%), which had been implanted from 0.2 to 4 years. They noted that the docking button of the device was inaccessible in 6 patients, and it had detached in 1 patient. No severe complications occurred after device retrieval; however, there were two instances of tricuspid valve damage [32]. Furthermore, long-term retrieval of Nanostim ${ }^{\mathrm{TM}}$ in 34 patients with an average implantation duration of 1,570 \pm 479 days was evaluated in a single-center study. The retrieval success rate was $85 \%$, with no procedure-related complications. The investigators noted that the retrieval success rate was significantly higher in patients with a more remarkable swinging movement of the docking button on fluoroscopic visualization compared to those without significant swinging. Consistent with previous studies, the docking button was not engageable in 5 patients as it was trapped by the surrounding tissue [77]. Early retrieval of Micra ${ }^{\mathrm{TM}}$ has been shown to be safe and feasible [74]; however, there is a paucity of data regarding long-term retrieval. Dar et al. [75] retrospectively compared the safety and efficacy of Nanostim ${ }^{\mathrm{TM}}$ and Micra ${ }^{\mathrm{TM}}$ retrieval. This study included 73 Nanostim $^{\mathrm{TM}}$ and $40 \mathrm{Mi}$ $\mathrm{cra}^{\mathrm{TM}}$ retrievals, with a median extraction time of 256 days and 46 days, respectively. The retrieval success rate for

Leadless Pacemakers: Current and Future Devices
Nanostim ${ }^{\mathrm{TM}}$ was $90 \%$ and $100 \%$ for Micra ${ }^{\mathrm{TM}}$. When comparing the devices, the higher success rate with Micra ${ }^{\mathrm{TM}}$ retrieval was attributed to the simple disengagement techniques, without reliance on the engagement of a proximal retrieval feature as seen with Nanostim ${ }^{\mathrm{TM}}$ [75].

\section{Conclusion}

Leadless pacemakers have emerged as a revolutionary innovation to mitigate the complications seen with transvenous pacemakers. Currently, Micra ${ }^{\mathrm{TM}}$ TPS or AV with VVI or VDD functionality is the sole leadless pacemakers approved for commercial use worldwide. Although this device has been promising, to date, there have been no randomized trials comparing leadless pacemakers to single chamber transvenous pacemakers. According to the recent 2021 European Society of Cardiology guidelines on cardiac pacing and CRT, leadless pacing should be considered as an alternative to standard transvenous pacemakers when there is an obstruction of the venous route used for standard pacemaker implantation or when the risk for device pocket infection is increased, such as in patients with previous pocket infections or those on hemodialysis [78].

Moreover, a leadless pacemaker with S-ICD system, implementing the novel modular cardiac rhythm management concept, demonstrated excellent feasibility in animal models; human studies are planned to start in the near future. Moreover, the WiSE-CRT ${ }^{\mathrm{TM}}$ provides a solution for patients unable to receive CRT due to coronary sinus anatomical constraints. However, this system is still prone to device- and lead-related complications. Therefore, data from ongoing trials will be imperative in establishing this new technology. With this device comes the prospect of a total leadless CRT system when combined with Micra ${ }^{\mathrm{TM}}$, which could usher in the new era in cardiac pacing. This combination system has shown efficacy in small studies; however, future trials assessing clinical safety and efficacy of this new system are needed.

\section{Acknowledgments}

I thank Dr. Thiago Gagliano-Jucá, $\mathrm{MD}, \mathrm{PhD}$, for providing editing assistance for the manuscript.

\section{Statement of Ethics}

Does not apply. 


\section{Conflict of Interest Statement}

J.G. declares that there is no conflict of interest.

\section{Funding Sources}

J.G. declares that there are no sources of funding.

\section{References}

1 Mond HG, Proclemer A. The 11th world survey of cardiac pacing and implantable cardioverter-defibrillators: calendar year 2009 - a world society of Arrhythmia's project. Pacing Clin Electrophysiol. 2011;34:1013-27.

2 Raatikainen MJP, Arnar DO, Zeppenfeld K, Merino JL, Levya F, Hindriks G, et al. Statistics on the use of cardiac electronic devices and electrophysiological procedures in the European Society of Cardiology countries: 2014 report from the European Heart Rhythm Association. Europace. 2015;17:11-75.

3 Larsson B, Elmqvist H, Rydén L, Schüller H. Lessons from the first patient with an implanted pacemaker: 1958-2001. Pacing Clin Electrophysiol. 2003;26:114-24.

4 Epstein AE, DiMarco JP, Ellenbogen KA, Estes NAM, Freedman RA, Gettes LS, et al. ACC/AHA/HRS 2008 Guidelines for devicebased therapy of cardiac rhythm abnormalities. A report of the American College of Cardiology/American Heart Association Task Force on practice guidelines (Writing Committee to revise the ACC/AHA/NASPE 2002 Guideline update for implantation of cardiac pacemakers and antiarrhythmia devices). J Am Coll Cardiol. 2008;51:e1-62.

5 ESC GUIDELINES 2013 ESC Guidelines on cardiac pacing and cardiac resynchronization therapy n.d.

6 Kusumoto FM, Schoenfeld MH, Barrett C, Edgerton JR, Ellenbogen KA, Gold MR, et al. ACC/AHA/HRS Guideline on the evaluation and management of patients with bradycardia and cardiac conduction delay: a report of the American College of Cardiology/American Heart Association Task Force on clinical practice guidelines and the Heart Rhythm Society. J Am Coll Cardiol. 2019;74:e51-156.

7 Kirkfeldt RE, Johansen JB, Nohr EA, Jørgensen OD, Nielsen JC. Complications after cardiac implantable electronic device implantations: an analysis of a complete, nationwide cohort in Denmark. Eur Heart J. 2014;35:1186-94.

8 Udo EO, Zuithoff NPA, Van Hemel NM, De Cock CC, Hendriks T, Doevendans PA, et al. Incidence and predictors of short- and longterm complications in pacemaker therapy: the FOLLOWPACE study. Hear Rhythm. 2012;9:728-35.

9 Kirkfeldt RE, Johansen JB, Nohr EA, Moller $M$, Arnsbo P, Nielsen JC. Pneumothorax in cardiac pacing: a population-based cohort study of 28860 Danish patients. Europace. 2012;14:1132-8.

10 Kiviniemi MS, Pirnes MA, Eränen HJ, Kettunen RV, Hartikainen JE. Complications related to permanent pacemaker therapy. Pacing Clin Electrophysiol. 1999;22:711-20.
11 Ellenbogen KA, Hellkamp AS, Wilkoff BL, Camunãs JL, Love JC, Hadjis TA, et al. Complications arising after implantation of DDD pacemakers: the MOST experience. Am J Cardiol. 2003;92:740-1.

12 Haghjoo M, Nikoo MH, Fazelifar AF, Alizadeh A, Emkanjoo Z, Sadr-Ameli MA. Predictors of venous obstruction following pacemaker or implantable cardioverter-defibrillator implantation: a contrast venographic study on 100 patients admitted for generator change, lead revision, or device upgrade. Europace. 2007;9:328-32.

13 Harcombe AA, Newell SA, Ludman PF, Wistow TE, Sharples LD, Schofield PM, et al. Late complications following permanent pacemaker implantation or elective unit replacement. Heart. 1998;80:240-4.

14 Tarakji KG, Wazni OM, Harb S, Hsu A, Saliba W, Wilkoff BL. Risk factors for 1-year mortality among patients with cardiac implantable electronic device infection undergoing transvenous lead extraction: the impact of the infection type and the presence of vegetation on survival. Europace. 2014;16:1490-5.

15 Birgersdotter-Green UM, Pretorius VG. Lead extractions: indications, procedural aspects, and outcomes. Cardiol Clin. 2014;32:201-10.

16 Maisel WH, Hauser RG, Hammill SC, Hauser RG, Ellenbogen KA, Epstein AE, et al. Recommendations from the Heart Rhythm Society Task Force on lead performance policies and guidelines. Developed in collaboration with the American College of Cardiology (ACC) and the American Heart Association (AHA). Hear Rhythm. 2009;6:869-85.

17 Spickler JW, Rasor NS, Kezdi P, Misra SN, Robins KE, LeBoeuf C. Totally self-contained intracardiac pacemaker. J Electrocardiol. 1970;3:325-31.

18 Vardas PE, Politopoulos C, Manios E, Parthenakis F, Tsagarkis C. A miniature pacemaker introduced intravenously and implanted endocardially. Preliminary findings from an experimental study. Eur J Card Pacing Electrophysiol. 1991;1:27-30. Available from: https: //scholar.google.com/scholar?clust er $=9975087670225174360 \& \quad \mathrm{hl}=\mathrm{en} \& \quad$ as $\mathrm{sdt}=400005 \&$ sciodt $=0$ Accessed 2021 May 2.

19 Wieneke H, Konorza T, Erbel R, Kisker E. Leadless pacing of the heart using induction technology: a feasibility study. Pacing Clin Electrophysiol. 2009;32:177-83.

20 Wieneke H, Rickers S, Velleuer J, Bruck G, Bai $\mathrm{Z}$, Kocks $\mathrm{C}$, et al. Leadless pacing using induction technology: Impact of pulse shape and geometric factors on pacing efficiency. Europace. 2013;15:453-9.
21 Koruth JS, Rippy MK, Khairkhahan A, Ligon DA, Hubbard CA, Goar FS, et al. Feasibility and efficacy of percutaneously delivered leadless cardiac pacing in an in vivo ovine model. J Cardiovasc Electrophysiol. 2015;26:322-8.

22 Reddy VY, Knops RE, Sperzel J, Miller MA, Petru J, Simon J, et al. Permanent leadless cardiac pacing: results of the LEADLESS trial. Circulation. 2014;129:1466-71.

23 Sperzel J, Burri H, Gras D, Tjong FVY, Knops RE, Hindricks G, et al. State of the art of leadless pacing. Europace. 2015;17:1508-13.

24 Merkel M, Grotherr P, Radzewitz A, Schmitt C. Leadless pacing: current state and future direction. Cardiol Ther. 2017;6:175-81.

25 Steinwender C, Lercher P, Schukro C, Blessberger H, Prenner G, Andreas M, et al. State of the art: leadless ventricular pacing - a national expert consensus of the Austrian Society of Cardiology. J Interv Card Electrophysiol. 2020;57:27-37.

26 Soejima K, Edmonson J, Ellingson ML, Herberg B, Wiklund C, Zhao J. Safety evaluation of a leadless transcatheter pacemaker for magnetic resonance imaging use. Heart Rhythm. 2016;13:2056-63.

27 Blessberger H, Kiblboeck D, Reiter C, Lambert T, Kellermair J, Schmit P, et al. Monocenter Investigation Micra ${ }^{\circledR}$ MRI study (MIMIC$\mathrm{RY}$ ): feasibility study of the magnetic resonance imaging compatibility of a leadless pacemaker system. Europace. 2019;21:13741.

28 Löbe S, Hilbert S, Hindricks G, Jahnke C, Paetsch I. Cardiovascular magnetic resonance imaging in a patient with implanted leadless pacemaker. JACC Clin Electrophysiol. 2018; 4:149-50.

29 Knops RE, Tjong FVY, Neuzil P, Sperzel J, Miller MA, Petru J, et al. Chronic performance of a leadless cardiac pacemaker: 1-year follow-up of the LEADLESS trial. J Am Coll Cardiol. 2015;65:1497-504.

30 Reddy VY, Exner DV, Cantillon DJ, Doshi R, Bunch TJ, Tomassoni GF, et al. Percutaneous implantation of an entirely intracardiac leadless pacemaker. N Engl J Med. 2015;373: 1125-35.

31 Sperzel J, Defaye P, Delnoy PP, Guerrero JJG, Knops RE, Tondo C, et al. Primary safety results from the LEADLESS observational study. Europace. 2018;20:1491-7.

32 Lakkireddy D, Knops R, Atwater B, Neuzil P, Ip J, Gonzalez E, et al. A worldwide experience of the management of battery failures and chronic device retrieval of the nanostim leadless pacemaker. Hear Rhythm. 2017;14:175663. 
33 Richter S, Döring M, Ebert M, Bode K, Müssigbrodt A, Sommer P, et al. Battery malfunction of a leadless cardiac pacemaker worrisome single-center experience. Circulation. 2018;137:2408-10.

34 Abbott Medical Devices. The LEADLESS II IDE Study (Phase II): a safety and effectiveness trial for a leadless pacemaker system. Available from: https://www.clinicaltrials. g o v / c t 2/ s h o w / r e c o r d / NCT04559945?term=NCT04559945\& draw=2\&rank=1 Accessed 2021 May 8.

35 Bonner M, Eggen M, Haddad T, Sheldon T, Williams E. Early performance and safety of the micra transcatheter pacemaker in pigs. Pacing Clin Electrophysiol. 2015;38:1248-59.

36 Nakashima T, Takaki H, Usami S, Yamada Y, Okamura H, Aiba T, et al. Multi-directional ventricular conduction on magnetocardiography predicts poor prognosis after CRT implantation assessment of leadless pacemaker performance. Eur Heart J. 2013;34:1917.

37 Reynolds D, Duray GZ, Omar R, Soejima K, Neuzil P, Zhang S, et al. A leadless intracardiac transcatheter pacing system. N Engl J Med. 2016;374:533-41.

38 Ritter P, Duray GZ, Zhang S, Narasimhan C, Soejima K, Omar R, et al. The rationale and design of the Micra Transcatheter Pacing Study: safety and efficacy of a novel miniaturized pacemaker. Europace. 2015;17:807-13.

39 Duray GZ, Ritter P, El-Chami M, Narasimhan C, Omar R, Tolosana JM, et al. Long-term performance of a transcatheter pacing system: 12-month results from the Micra Transcatheter Pacing Study. Hear Rhythm. 2017;14: 702-9.

40 Roberts PR, Clementy N, Al Samadi F, Garweg C, Martinez-Sande JL, Iacopino S, et al. A leadless pacemaker in the real-world setting: the Micra Transcatheter Pacing System PostApproval Registry. Hear Rhythm. 2017;14: 1375-9.

41 Medtronic. Longitudinal coverage with Evidence Development Study on micra leadless pacemakers. Available from: https://clinicaltrials.gov/ct2/show/record/NCT03039712 Accessed 2021 May 8.

42 Greenspon AJ, Patel JD, Lau E, Ochoa JA, Frisch DR, Ho RT, et al. Trends in permanent pacemaker implantation in the United States from 1993 to 2009: increasing complexity of patients and procedures. J Am Coll Cardiol. 2012;60:1540-5.

43 Nielsen JC, Andersen HR, Thomsen PEB, Thuesen L, Mortensen PT, Vesterlund T, et al. Heart failure and echocardiographic changes during long-term follow-up of patients with sick sinus syndrome randomized to singlechamber atrial or ventricular pacing. Circulation. 1998;97:987-95.

44 Lamas GA, Orav EJ, Stambler BS, Ellenbogen KA, Sgarbossa EB, Huang SKS, et al. Quality of life and clinical outcomes in elderly patients treated with ventricular pacing as compared with dual-chamber pacing. N Engl J Med. 1998;338:1097-104.
45 Lamas GA, Ellenbogen KA, Hennekens CH, Montanez A. Evidence base for pacemaker mode selection: from physiology to randomized trials. Circulation. 2004;109(4):443-51.

46 Chinitz L, Ritter P, Khelae SK, Iacopino S, Garweg C, Grazia-Bongiorni M, et al. Accelerometer-based atrioventricular synchronous pacing with a ventricular leadless pacemaker: results from the Micra atrioventricular feasibility studies. Hear Rhythm. 2018;15:136371.

47 Steinwender C, Khelae SK, Garweg C, Chan JYS, Ritter P, Johansen JB, et al. Atrioventricular synchronous pacing using a leadless ventricular pacemaker: results from the MARVEL 2 Study. JACC Clin Electrophysiol. 2020; 6:94-106.

48 Tjong FVY, Brouwer TF, Kooiman KM, Smeding L, Koop B, Soltis B, et al. Communicating antitachycardia pacing-enabled leadless pacemaker and subcutaneous implantable defibrillator. J Am Coll Cardiol. 2016;67: 1865-6.

49 Bardy GH, Smith WM, Hood MA, Crozier IG, Melton IC, Jordaens L, et al. An entirely subcutaneous implantable cardioverter-defibrillator. N Engl J Med. 2010;363:36-44.

50 Weiss R, Knight BP, Gold MR, Leon AR, Herre JM, Hood M, et al. Safety and efficacy of a totally subcutaneous implantable-cardioverter defibrillator. Circulation. 2013;128: 944-53.

51 Burke MC, Gold MR, Knight BP, Barr CS, Theuns DAMJ, Boersma LVA, et al. Safety and efficacy of the totally subcutaneous implantable defibrillator: 2-year results from a pooled analysis of the IDE study and EFFORTLESS registry. J Am Coll Cardiol. 2015; 65:1605-15.

52 Tjong FVY, Brouwer TF, Koop B, Soltis B, Shuros A, Schmidt B, et al. Acute and 3-month performance of a communicating leadless antitachycardia pacemaker and subcutaneous implantable defibrillator. JACC Clin Electrophysiol. 2017;3:1487-98.

53 Tjong FVY, Koop BE. The modular cardiac rhythm management system: the EMPOWER leadless pacemaker and the EMBLEM subcutaneous ICD. Herzschrittmacherther Elektrophysiol. 2018;29:355-61.

54 Moss AJ, Hall WJ, Cannom DS, Klein H, Brown MW, Daubert JP, et al. Cardiac-resynchronization therapy for the prevention of heart-failure events. N Engl J Med. 2009;361: 1329-38.

55 Nelson GS, Berger RD, Fetics BJ, Talbot M, Hare JM, Kass DA, et al. Left ventricular or biventricular pacing improves cardiac function at diminished energy cost in patients with dilated cardiomyopathy and left bundlebranch block. Circulation. 2000;102:3053-9.

56 Cleland JGF, Daubert JC, Erdmann E, Freemantle N, Gras D, Kappenberger L, et al. The effect of cardiac resynchronization on morbidity and mortality in heart failure. $\mathrm{N}$ Engl J Med. 2005;352:1539-49.
57 McAlister FA, Ezekowitz J, Hooton N, Vandermeer B, Spooner C, Dryden DM, et al. Cardiac resynchronization therapy for patients with left ventricular systolic dysfunction: a systematic review. J Am Med Assoc. 2007;297: 2502-14.

58 León AR, Abraham WT, Curtis AB, Daubert JP, Fisher WG, Gurley J, et al. Safety of transvenous cardiac resynchronization system implantation in patients with chronic heart failure: combined results of over 2,000 patients from a multicenter study program. J Am Coll Cardiol. 2005;46:2348-56.

59 Gamble JHP, Herring N, Ginks M, Rajappan K, Bashir Y, Betts TR. Procedural success of left ventricular lead placement for cardiac resynchronization therapy: a metaanalysis. JACC Clin Electrophysiol. 2016; 2(1):69-77.

60 Foley PW, Leyva F, Frenneaux MP. What is treatment success in cardiac resynchronization therapy? Europace. 2009;11(Suppl 5): v58-65.

61 Lee KL, Lau CP, Tse HF, Echt DS, Heaven D, Smith W, et al. First human demonstration of cardiac stimulation with transcutaneous ultrasound energy delivery. Implications for wireless pacing with implantable devices. J Am Coll Cardiol. 2007;50:877-83.

62 Lee KL, Tse HF, Echt DS, Lau CP. Temporary leadless pacing in heart failure patients with ultrasound-mediated stimulation energy and effects on the acoustic window. Heart Rhythm. 2009;6:742-8.

63 Auricchio A, Delnoy PP, Regoli F, Seifert M, Markou T, Butter C. First-in-man implantation of leadless ultrasound-based cardiac stimulation pacing system: novel endocardial left ventricular resynchronization therapy in heart failure patients. Europace. 2013;15: 1191-7.

64 Sieniewicz BJ, Gould JS, Rimington HM, Ioannou N, Rinaldi CA. Transseptal delivery of a leadless left ventricular endocardial pacing electrode. JACC Clin Electrophysiol. 2017;3: $1333-5$.

65 Reddy VY, Miller MA, Neuzil P, Søgaard P, Butter C, Seifert M, et al. Cardiac resynchronization therapy with wireless left ventricular endocardial pacing: the SELECT-LV Study. J Am Coll Cardiol. 2017;69:2119-29.

66 Auricchio A, Delnoy PP, Butter C, Brachmann J, Van Erven L, Spitzer S, et al. Feasibility, safety, and short-term outcome of leadless ultrasound-based endocardial left ventricular resynchronization in heart failure patients: results of the wireless stimulation endocardially for CRT (WiSE-CRT) study. Europace. 2014;16:681-8.

67 Sieniewicz BJ, Betts TR, James S, Turley A, Butter C, Seifert M, et al. Real-world experience of leadless left ventricular endocardial cardiac resynchronization therapy: a multicenter international registry of the WiSECRT pacing system. Hear Rhythm. 2020;17: 1291-7. 
68 Singh JP, Abraham WT, Auricchio A, Delnoy PP, Gold M, Reddy VY, et al. Design and rationale for the stimulation of the left ventricular endocardium for cardiac resynchronization therapy in non-responders and previously untreatable patients (SOLVE-CRT) trial. Am Heart J. 2019;217:13-22.

69 Montemerlo E, Pozzi M, De Ceglia S, Santini F, Piazzi E, Rovaris G. First-in-man fully leadless transvenous CRT-P with a transseptal implant of WISE-CRT ${ }^{\circ}$ system and Micra ${ }^{\circ} \mathrm{PM}$. Pacing Clin Electrophysiol. 2019;42:1489-92.

70 Funasako M, Neuzil P, Dujka L, Petru J, Sediva L, Simon J, et al. Successful implementation of a totally leadless biventricular pacing approach. Hear Case Reports. 2020;6:153-7.

71 Carabelli A, Jabeur M, Jacon P, Rinaldi CA, Leclercq C, Rovaris G, et al. European experience with a first totally leadless cardiac resynchronization therapy pacemaker system. Eurospace. 2021;23:740-7.
72 Sidhu BS, Gould J, Porter B, Elliott M, Mehta V, Niederer S, et al. Completely leadless cardiac resynchronization defibrillator system. JACC Clin Electrophysiol. 2020;6:588-9.

73 Li J, Hou WB, Cao MK, Zhou WX, Wang Y, Fang Y, et al. Safety and efficacy of leadless pacemaker retrieval. J Cardiovasc Electrophysiol. 2019;30:1671-8.

74 Afzal MR, Daoud EG, Cunnane R, Mulpuru SK, Koay A, Hussain A, et al. Techniques for successful early retrieval of the Micra transcatheter pacing system: a worldwide experience. Hear Rhythm. 2018;15:841-6.

75 Dar T, Akella K, Murtaza G, Sharma S, Afzal MR, Gopinathannair R, et al. Comparison of the safety and efficacy of Nanostim and Micra transcatheter leadless pacemaker (LP) extractions: a multicenter experience. J Interv Card Electrophysiol. 2020;57:133-40.
76 Reddy VY, Miller MA, Knops RE, Neuzil P, Defaye P, Jung W, et al. Retrieval of the leadless cardiac pacemaker: a multicenter experience. Circ Arrhythm Electrophysiol. 2016;9.

77 Minami K, Neužil P, Petrů J, Chovanec M, Hubbard C, Hála P, et al. Retrieval of longterm implanted leadless pacemakers: a singlecenter experience. JACC Clin Electrophysiol. 2020;6:1744-51.

78 Glikson M, Nielsen JC, Kronborg MB, Michowitz Y, Auricchio A, Barbash IM, et al. 2021 ESC Guidelines on cardiac pacing and cardiac resynchronization therapy developed by the task force on cardiac pacing and cardiac resynchronization therapy of the European Society of Cardiology (ESC) with the special contribution of the European Heart Rhythm Association (EHRA). Eur Heart J. 2021;42:3427-520. 